ANTICANCER THERAPY

\section{Complementing tumour macrophage reprogramming}

\begin{abstract}
As one of the main pathways in innate immunity, complement cascades are involved in recognition and elimination of pathogens and damaged cells. However, sustained complement activation is associated with chronic inflammation and tumour development. Now, writing in Cancer Cell, Medler et al. report the role of complement C5a in promoting an immune-suppressive tumour microenvironment in squamous cell carcinoma (SCC). Blockade of the C5a receptor, C5aR1, can reprogramme tumour-associated macrophages (TAMs) from an M2 protumorigenic state to an M1 antitumour state, offering a new immunomodulatory strategy.

Chronic activation of inflammatory pathways drives tumour progression in SCC, so the authors first hypothesized whether generation of complement might be involved in this process. Using a transgenic mouse model of epithelial squamous cell carcinogenesis, they observed abundant deposition of $\mathrm{C} 5 \mathrm{a}$ and increased
\end{abstract}

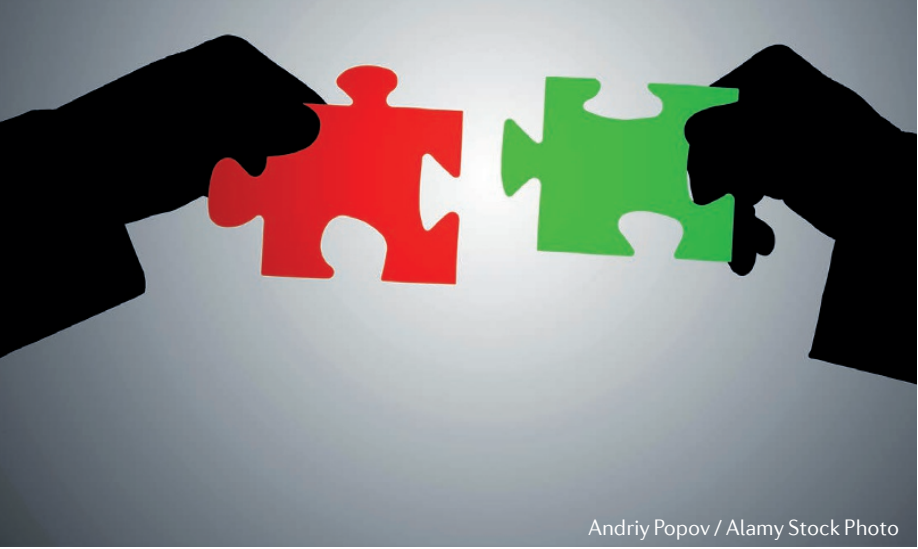

presence of $\mathrm{C} 5 \mathrm{aR} 1^{+}$cells in areas adjacent to premalignant dysplasias, indicating that $\mathrm{C} 5 \mathrm{a}$ deposition is an early feature of squamous carcinogenesis. C5aR1 $1^{+}$cells were identified as mast cells and $\mathrm{F} 4 / 80^{+}$macrophages, among other leukocytes.

Orthotopic growth of the SCC cell line, PDSC5, was considerably restricted in $\mathrm{C} 5 \mathrm{aR} 1^{-1-}$ mice and the authors wondered whether it was C5aR1 expression in mast cells and macrophages that was needed to drive tumorigenesis. Indeed, coinjection of $\mathrm{C} \mathrm{aR} 1^{+/}$bone marrowderived mast cells or macrophages with PDSC5 cells restored tumour growth kinetics in $\mathrm{C} 5 \mathrm{aR} 1^{-/-}$mice to levels observed in wild-type mice, whereas co-injection with $\mathrm{C} 5 \mathrm{aR} 1^{-1}$ mast cells or macrophages did not. A retrospective analysis of a large data set of human SCC biopsy samples showed high infiltration of $\mathrm{C} 5 \mathrm{aR} 1^{+}$leukocytes and a significant inverse correlation between survival and intratumoural C5aR1 expression.

Several members of the coagulation and fibrinolytic cascade generate $\mathrm{C} 5 \mathrm{a}$ but plasmin seemed a likely candidate in this setting, as it is activated by urokinase plasminogen activator (uPA), a serine protease that is highly expressed in several human solid tumours. As observed in $\mathrm{C}_{5} \mathrm{aR} 1^{-/-}$ mice, tumour growth of PDSC5 cells was considerably slower in $\mathrm{uPA}^{-/-}$mice compared with $\mathrm{uPA}^{+-}$mice. Further experiments showed that plasminogen processing was a property of pro-tumorigenic M2-TAMs.

To assess whether C5aR1 represents a therapeutic target, Medler et al. tested the ability of a $\mathrm{C} 5 \mathrm{aR} 1$ peptide antagonist, PMX-53 - a compound previously developed for the treatment of psoriasis and ocular indications such as age-related macular degeneration - to inhibit the growth of orthotopic SCC implants in combination with the chemotherapy drug paclitaxel (PTX). Whereas monotherapy with either PMX53 or PTX did not alter tumour growth, combination therapy was synergistically effective in slowing tumour growth even at late stages of tumour development. Analysis of the tumours in treated mice showed that TAMs expressed gene signatures associated with interferon (IFN) $-\gamma$ responses and higher levels of several mRNAs associated with induction of Thelper 1-type responses as well as Cxcl9 and Cxcl11, which are IFN $\gamma$ responsive chemokines that recruit cytotoxic CXCR $3^{+} \mathrm{CD}^{+} \mathrm{T}$ cells.

Depletion of $\mathrm{CD}^{+} \mathrm{T}$ cells restored vascular density and tumour growth in treated mice, showing that these cells were required for efficacy of the combination therapy. Similarly, both blockade of IFN $\gamma$ and reduction of macrophage presence with a neutralizing monoclonal antibody against colony-stimulating factor 1 (CSF1) reversed treatment efficacy and reduced homing of CXCR $3^{+}$ $\mathrm{CD}^{+} \mathrm{T}$ cells. The authors concluded that $\mathrm{CXCR}^{+} \mathrm{CD}^{+} \mathrm{T}$ cells regulate the response to chemotherapy by infiltrating the tumours following IFN $\gamma$-induced TAM reprogramming to an antitumour phenotype.

These data present therapeutic inhibition of C5aR1 as a new immunomodulatory strategy to harness the adaptive immune system to achieve an antitumour response, an approach that might be particularly helpful in patients with SCCs that are highly infiltrated with $\mathrm{C} 5 \mathrm{aR} 1^{+}$cells.

M. Teresa Villanueva

ORIGINAL ARTICLE Medler, T. R. et al. Complement $\mathrm{C} 5$ a fosters squamous carcinogenesis and limits $T$ cell responses to chemotherapy. Cancer Cell 34, 561-578 (2018) FURTHER READING Cassetta, L. \& Pollard, J. Targeting macrophages: therapeutic approaches in cancer. Nat. Rev. Drug. Discov. https://doi. org/10.1038/nrd.2018.169 (2018) 